\title{
A Study on Challenges faced by Mothers and the Coping Strategies adopted by them during the Postpartum Period
}

\author{
${ }^{1}$ Sabitha Nayak, ${ }^{2}$ Prasanna K Shetty
}

\begin{abstract}
Postnatal period begins immediately after the birth of the newborn and extends up to 6 weeks. It is significant for the mother, for the baby, and for the family as it is a time of physiological adjustment for the mother and the baby and emotional and social adjustment for all involved in the care. A descriptive study was conducted among 500 mothers on challenges faced by mothers and the coping strategies adopted by them during the postpartum period at Justice K.S. Hegde Hospital, Mangaluru, Karnataka, India, by purposive sampling, and the study results indicated that almost all the mothers had a good adaptation toward motherhood.
\end{abstract}

Keywords: Coping strategies, Mothers, Postpartum challenges.

How to cite this article: Nayak S, Shetty PK. A Study on Challenges faced by Mothers and the Coping Strategies adopted by them during the Postpartum Period. J South Asian Feder Obst Gynae 2017;9(4):348-355.

Source of support: Nil

Conflict of interest: None

Date of received: 10 October 2017

Date of acceptance: 1 December 2017

Date of publication: January 2018

\section{INTRODUCTION}

Postnatal period begins immediately after the birth of the newborn and extends up to 6 weeks. It is significant for the mother, for the baby, and for the family as it is a time of physiological adjustment for the mother and the baby and emotional and social adjustment for all involved in the care. During this period, the woman has to make major adjustments as this may in turn be crucial for her present and future ability to function. This period is also the challenging period in her reproductive life. The transition from a nonmother to a mother, or from being a mother of one child to becoming a mother of two children, calls for changes in a woman's role relationships

\footnotetext{
${ }^{1,2}$ Professor

${ }^{1}$ Department of Obstetrics and Gynecology Nursing, Nitte Usha Institute of Nursing Sciences, Mangaluru, Karnataka, India

${ }^{2}$ Department of Obstetrics and Gynecology, K.S. Hegde Medical Academy, Mangaluru, Karnataka, India

Corresponding Author: Sabitha Nayak, Professor, Department of Obstetrics and Gynecology Nursing, Nitte Usha Institute of Nursing Sciences, Mangaluru, Karnataka, India, Phone: +919448842698, e-mail: sabitha_nayak@yahoo.ie
}

and/or alteration in her behavior and in herself in the social context. ${ }^{1-4}$

\section{OBJECTIVES}

- To determine the challenges faced by postpartum mothers

- To find the coping strategies adopted by them,

- To find an association between challenges faced by postpartum women with selected demographic variables,

- To find an association between coping strategies adopted by postpartum women with selected demographic variables, and

- To compare the differences between challenges faced by primi-mothers and multimothers, and their coping strategies.

\section{MATERIALS AND METHODS}

A quantitative descriptive survey design was utilized, and the sample size was 500 postnatal mothers which was through purposive sampling technique in postnatal wards of the Department of OBG at Justice K.S. Hegde Hospital by using structured rating scale for postpartum challenges and for coping strategies. Structured checklist of 25 items was used.

\section{RESULTS}

The demographic characteristics were as follows: $45.8 \%$ were in the age group of 25 to 29 years and $2 \%$ were in age group of 18 to 20 years. Most of the participants were of Hindu religion (66.4\%) (Table 1). Regarding literacy, $39 \%$ studied up to high school and only $0.4 \%$ were Christians. Most of the participants were nonworking (84.6\%). Regarding family income, $35.4 \%$ were between 5,001 and $10,000 \mathrm{INR}$, and $22 \%$ were having an income of more than Rs. 15,000 . About $53.2 \%$ had more than one child, and $46.8 \%$ had one child. Most of the participants had normal vaginal delivery $(62 \%)$. With regard to gestational age at delivery, $29.2 \%$ delivered at 39 weeks and $4.2 \%$ more than 40 weeks. $52.8 \%$ delivered male baby and $47.2 \%$ female baby. Regarding weight of the baby, in $81.4 \%$, the baby weighed more than $2.5 \mathrm{~kg}$. With regard to physiological challenges, most of the mothers had positive adaptation. About $34.2 \%$ had pain at the suture site sometimes, and 


\begin{tabular}{|c|c|c|c|}
\hline & & Frequency & Percentage \\
\hline \multirow[t]{5}{*}{ Age (in years) } & $18-20$ & 10 & 2 \\
\hline & $21-24$ & 155 & 31 \\
\hline & $25-29$ & 229 & 45.8 \\
\hline & $30-34$ & 84 & 16.8 \\
\hline & 35 and above & 22 & 4.4 \\
\hline \multirow[t]{3}{*}{ Religion } & Hindu & 332 & 66.4 \\
\hline & Muslim & 152 & 30.4 \\
\hline & Christian & 16 & 3.2 \\
\hline \multirow[t]{6}{*}{ Educational status } & Illiterate & 2 & 0.4 \\
\hline & $1-7$ & 115 & 23 \\
\hline & $8-10$ & 195 & 39 \\
\hline & PUC & 127 & 25.4 \\
\hline & Degree & 58 & 11.6 \\
\hline & Postgraduation & 3 & 0.6 \\
\hline \multirow[t]{2}{*}{ Occupation } & Working & 77 & 15.4 \\
\hline & Nonworking & 423 & 84.6 \\
\hline \multirow[t]{4}{*}{ Family income } & $\leq 5,000$ INR & 117 & 23.4 \\
\hline & $5,001-10,000$ INR & 177 & 35.4 \\
\hline & $10,001-15,000$ INR & 96 & 19.2 \\
\hline & $>15,000$ INR & 110 & 22 \\
\hline \multirow[t]{2}{*}{ Parity } & 1 & 234 & 46.8 \\
\hline & 2 and more & 266 & 53.2 \\
\hline \multirow[t]{3}{*}{ Type of delivery } & Normal & 310 & 62 \\
\hline & Cesarean & 187 & 37.4 \\
\hline & Others & 3 & 0.6 \\
\hline \multirow[t]{5}{*}{ Gestational age } & 37 weeks & 97 & 19.4 \\
\hline & 38 weeks & 99 & 19.8 \\
\hline & 39 weeks & 146 & 29.2 \\
\hline & 40 weeks & 137 & 27.4 \\
\hline & More than 40 weeks & 21 & 4.2 \\
\hline \multirow[t]{2}{*}{ Sex of the baby } & Male & 264 & 52.8 \\
\hline & Female & 236 & 47.2 \\
\hline \multirow[t]{2}{*}{ Weight of the baby } & $<2.5 \mathrm{~kg}$ & 93 & 18.6 \\
\hline & $\geq 2.5 \mathrm{~kg}$ & 407 & 81.4 \\
\hline
\end{tabular}

$14.2 \%$ of the participants never felt pain. About $36.8 \%$ were fatigued sometimes and $36.8 \%$ were never able to rest comfortably. About $28.8 \%$ had sleep disturbance sometimes and $51.4 \%$ had no breast engorgement. About $50.8 \%$ never had abdominal pain after delivery. Under emotional challenges, most of the mothers were able to cope up positively with challenges and had good adaptation, and some participants were unable to adapt positively. About $87.8 \%$ of mothers never had persistent feelings of not being a good mother. About $64 \%$ of mothers were not depressed due to exhaustion. All the mothers had positive feeling toward the sex of the baby. About $72 \%$ were not anxious at all always, and $76.4 \%$ of the mothers were joyful. About $74 \%$ of the mothers were never depressed due to the physical changes of pregnancy and childbirth. About $89 \%$ were never disturbed by the sex of the baby. About $74 \%$ had positive feeling toward sex of the baby. With regard to environmental challenges, $55 \%$ were not distressed with the physical setup and only $10.2 \%$ felt most of the time the environment was distressing. About $56.2 \%$ never felt that visitors were a hindrance. Only $4.6 \%$ felt that there was noise in the ward always. Only $25.6 \%$ felt that privacy was maintained always, whereas $36.4 \%$ felt that privacy was never maintained, and only $14.8 \%$ felt that privacy was maintained most of the time. Only $85.2 \%$ were extremely happy with breastfeeding, whereas only $2 \%$ were never happy with breastfeeding. 56\% had no latching difficulty; $24 \%$ expected breastfeeding assistance sometimes and $44.8 \%$ never expected breastfeeding assistance. About $89.8 \%$ of mothers were never reluctant to 
feed. With regard to communication challenges, $52.2 \%$ expressed that all the health professionals communicated to them always and $6.4 \%$ felt that all the health professionals never communicated with them. About $37.8 \%$ felt that proper communication by the midwives and student midwives eased their discomfort always, and 13\% felt that student midwives were involved in their care always and $27.4 \%$ sometimes. Regarding newborn challenges, $73.2 \%$ were never irritated when the baby was crying and only $5.2 \%$ were irritated always when the baby was crying. About $31.8 \%$ were never aware of the newborn care and $35.4 \%$ were totally aware of the newborn care always. About $30 \%$ of mothers never had the difficulty in pacifying the elder sibling. Regarding employment, $75.6 \%$ of the participants were homemakers. Regarding family support, $71.2 \%$ of the mothers felt their family members were supportive always and only $4.8 \%$ felt never supportive.
About $73.2 \%$ felt that their in-laws were caring always and 93.4\% felt that their spouse was caring always. Astructured checklist with yes / no categories was used to assess coping strategies, and it was found that almost all the mothers were able to cope up well. About $98.8 \%$ expressed that they were able to console the baby appropriately when the baby was crying. About $94.8 \%$ were able to take care of the child and $94.4 \%$ did not react hastily or emotionally. There was significant association between physiological challenges and selected demographic variables, such as occupation $(0.061, \mathrm{p}<0.05)$ and family income (0.001, p < 0.05). Regarding association with breastfeeding and selected demographic variables, such as age $(0.074$, $\mathrm{p}<0.05)$, educational status $(0.024, \mathrm{p}<0.05)$, family income $(0.021)$, parity (0.000), gestational age $(0.026)$, and sex of the baby $(0.087, \mathrm{p}<0.05)$, there was significant association. The data presented in Table 2 shows that there is association

Table 2: Association between breastfeeding challenges and demographic variables $(n=500)$

\begin{tabular}{|c|c|c|c|c|c|}
\hline \multirow[b]{2}{*}{ Demographics } & & \multicolumn{2}{|c|}{ Breastfeeding challenges } & \multicolumn{2}{|c|}{ Chi-square } \\
\hline & & smedian (10) & >median (10) & Value & $p$-value \\
\hline \multirow[t]{5}{*}{ Age (in years) } & $18-20$ & 7 & 3 & 8.463 & $0.074 p<0.05 S$ \\
\hline & $21-24$ & 94 & 61 & & \\
\hline & $25-29$ & 118 & 111 & & \\
\hline & $30-34$ & 36 & 48 & & \\
\hline & 35 and above & 12 & 10 & & \\
\hline \multirow[t]{3}{*}{ Religion } & Hindu & 195 & 137 & 11.505 & $0.003 p<0.05 S$ \\
\hline & Muslim & 66 & 86 & & \\
\hline & Christian & 6 & 10 & & \\
\hline \multirow[t]{6}{*}{ Educational status } & Illiterate & 1 & 1 & 11.659 & $0.024 p<0.05 S$ \\
\hline & $1-7$ & 47 & 68 & & \\
\hline & $8-10$ & 109 & 86 & & \\
\hline & PUC & 74 & 53 & & \\
\hline & Degree & 33 & 25 & & \\
\hline & Postgraduation & 3 & 0 & & \\
\hline \multirow[t]{2}{*}{ Occupation } & Working & 46 & 31 & 1.485 & $0.633 p>0.05 \mathrm{NS}$ \\
\hline & Nonworking & 221 & 202 & & \\
\hline \multirow[t]{4}{*}{ Family income } & $\leq 5,000$ INR & 67 & 50 & 9.698 & $0.021 p<0.05 S$ \\
\hline & $5,001-10,000$ INR & 81 & 96 & & \\
\hline & $10,001-15,000$ INR & 49 & 47 & & \\
\hline & $>15,000$ INR & 70 & 40 & & \\
\hline \multirow[t]{2}{*}{ Parity } & 1 & 159 & 75 & 39.498 & $0.000 p<0.05 S$ \\
\hline & 2 and more & 109 & 158 & & \\
\hline \multirow[t]{3}{*}{ Type of delivery } & Normal & 158 & 152 & 2.891 & $0.410 p>0.05 \mathrm{NS}$ \\
\hline & Cesarean & 107 & 80 & & \\
\hline & Others & 2 & 1 & & \\
\hline \multirow{5}{*}{ Gestational age } & 37 weeks & 58 & 39 & 10.985 & $0.026 p<0.05 S$ \\
\hline & 38 weeks & 54 & 45 & & \\
\hline & 39 weeks & 63 & 83 & & \\
\hline & 40 weeks & 77 & 60 & & \\
\hline & More than 40 weeks & 15 & 6 & & \\
\hline \multirow[t]{2}{*}{ Sex of the baby } & Male & 130 & 134 & 4.177 & $0.087 p<0.05 S$ \\
\hline & Female & 137 & 99 & & \\
\hline \multirow[t]{2}{*}{ Weight of the baby } & $<2.5 \mathrm{~kg}$ & 47 & 46 & 0.376 & $0.566 p>0.05 \mathrm{NS}$ \\
\hline & $\geq 2.5 \mathrm{~kg}$ & 220 & 187 & & \\
\hline
\end{tabular}

S: Significant; NS: Not significant 
A Study on Challenges faced by Mothers and them Coping Strategies

Table 3: Association between emotional challenges and demographic variables $(n=500)$

\begin{tabular}{|c|c|c|c|c|c|}
\hline \multirow{2}{*}{ Demographics } & & \multicolumn{2}{|c|}{ Breastfeeding challenges } & \multicolumn{2}{|c|}{ Chi-square } \\
\hline & & $\leq$ median (57) & >median (57) & Value & $p$-value \\
\hline \multirow[t]{5}{*}{ Age (in years) } & $18-20$ & 7 & 3 & 4.162 & $0.389 p>0.05 \mathrm{NS}$ \\
\hline & $21-24$ & 83 & 72 & & \\
\hline & $25-29$ & 133 & 96 & & \\
\hline & $30-34$ & 45 & 39 & & \\
\hline & 35 and above & 16 & 6 & & \\
\hline \multirow[t]{3}{*}{ Religion } & Hindu & 189 & 143 & 2.460 & $0.312 p>0.05 \mathrm{NS}$ \\
\hline & Muslim & 83 & 69 & & \\
\hline & Christian & 12 & 4 & & \\
\hline \multirow[t]{6}{*}{ Educational status } & Illiterate & 1 & 1 & 8.063 & $0.120 p>0.05 \mathrm{NS}$ \\
\hline & $1-7$ & 61 & 54 & & \\
\hline & $8-10$ & 103 & 92 & & \\
\hline & PUC & 76 & 51 & & \\
\hline & Degree & 40 & 18 & & \\
\hline & Postgraduation & 3 & 0 & & \\
\hline \multirow[t]{2}{*}{ Occupation } & Working & 47 & 30 & 2.823 & $0.204 p>0.05 \mathrm{NS}$ \\
\hline & Nonworking & 237 & 186 & & \\
\hline \multirow[t]{4}{*}{ Family income } & $\leq 5,000$ INR & 80 & 37 & 12.010 & $0.007 p<0.05 S$ \\
\hline & $5,001-10,000$ INR & 93 & 84 & & \\
\hline & 10,001-15,000 INR & 45 & 51 & & \\
\hline & $>15,000$ INR & 66 & 44 & & \\
\hline \multirow[t]{2}{*}{ Parity } & 1 & 138 & 96 & 2.917 & $0.409 p>0.05 \mathrm{NS}$ \\
\hline & 2 and more & 146 & 120 & & \\
\hline \multirow[t]{3}{*}{ Type of delivery } & Normal & 158 & 152 & 12.312 & $0.002 p<0.05 S$ \\
\hline & Cesarean & 123 & 64 & & \\
\hline & Others & 3 & 0 & & \\
\hline \multirow[t]{5}{*}{ Gestational age } & 37 weeks & 57 & 40 & 9.113 & $0.058 p<0.05 S$ \\
\hline & 38 weeks & 61 & 38 & & \\
\hline & 39 weeks & 70 & 76 & & \\
\hline & 40 weeks & 80 & 57 & & \\
\hline & More than 40 weeks & 16 & 5 & & \\
\hline \multirow[t]{2}{*}{ Sex of the baby } & Male & 139 & 125 & 4.752 & $0.060 p>0.05 S$ \\
\hline & Female & 145 & 91 & & \\
\hline \multirow[t]{2}{*}{ Weight of the baby } & $<2.5 \mathrm{~kg}$ & 55 & 38 & 0.255 & $0.644 p>0.05 \mathrm{NS}$ \\
\hline & $\geq 2.5 \mathrm{~kg}$ & 229 & 178 & & \\
\hline
\end{tabular}

S: Significant; NS: Not significant

with breastfeeding and selected demographic variables, such as age, religion, educational status, family income, parity, gestational age, and sex of the baby $(\mathrm{p}<0.05)$. There was significant association between communication challenges and coping strategies $(0.017, \mathrm{p}<0.05)$. There was significant association between emotional challenges and selected demographic variables, such as family income $(0.007, \mathrm{p}<0.05)$, type of delivery $(0.002, \mathrm{p}<0.05)$, gestational age $(0.058, \mathrm{p}<0.05)$, and sex of the baby $(0.060, \mathrm{p}<0.05)$. Table 3 depicts there was significant association between emotional challenges and selected demographic variables, such as family income, type of delivery, gestational age, and sex of the baby. There was significant association between employment challenges and selected demographic variables, such as educational status $(0.000, p<0.05)$, occupation $(0.000, \mathrm{p}<0.05)$, and parity $(0.000, \mathrm{p}<0.05)$. There was significant association between environmental challenges and selected demographic variables, such as educational status $(0.029, \mathrm{p}<0.05)$ and type of delivery $(0.035, \mathrm{p}<0.05)$.
Table 4 depicts there was significant association between environmental challenges and selected demographic variables, such as educational status and type of delivery. There was significant association between newborn challenges and selected demographic variables, such as age $(0.000$, $\mathrm{p}<0.05)$, educational status $(0.026, \mathrm{p}<0.05)$, occupation $(0.024, \mathrm{p}<0.05)$, and parity $(0.000, \mathrm{p}<0.05)$. Table 5 depicts there was significant association between newborn challenges and selected demographic variables, such as age, educational status, occupation, religion, and parity. Table 6 depicts there was significant association between physiological challenges and selected demographic variables, such as family income and occupation. Table 7 depicts that there was significant association between coping stratergies and demographic variables, such as occupation ( $p$-value $=0.020 ; p<0.05 ; S)$ and gestational age ( $p$-value $=0.04 ; \mathrm{p}<0.05 ; \mathrm{S})$. Table 8 depicts that there is difference in the mean in the areas, such as breastfeeding challenges, newborn challenges, and employment status. 
Table 4: Association between environmental challenges and demographic variables $(n=500)$

\begin{tabular}{|c|c|c|c|c|c|}
\hline \multirow{2}{*}{ Demographics } & & \multicolumn{2}{|c|}{ Environmental challenges } & \multicolumn{2}{|c|}{ Chi-square } \\
\hline & & $\leq$ median (13) & >median (13) & Value & $p$-value \\
\hline \multirow[t]{5}{*}{ Age (in years) } & $18-20$ & 6 & 4 & 5.414 & $0.249 p>0.05 \mathrm{NS}$ \\
\hline & $21-24$ & 85 & 70 & & \\
\hline & $25-29$ & 112 & 117 & & \\
\hline & $30-34$ & 43 & 41 & & \\
\hline & 35 and above & 16 & 6 & & \\
\hline \multirow[t]{3}{*}{ Religion } & Hindu & 176 & 156 & 0.541 & $0.791 p>0.05 \mathrm{NS}$ \\
\hline & Muslim & 79 & 73 & & \\
\hline & Christian & 7 & 9 & & \\
\hline \multirow[t]{6}{*}{ Educational status } & Illiterate & 2 & 0 & 11.237 & $0.029 p<0.05 S$ \\
\hline & $1-7$ & 50 & 65 & & \\
\hline & $8-10$ & 105 & 90 & & \\
\hline & PUC & 68 & 59 & & \\
\hline & Degree & 37 & 21 & & \\
\hline & Postgraduation & 0 & 3 & & \\
\hline \multirow[t]{2}{*}{ Occupation } & Working & 37 & 40 & 0.947 & $0.729 p>0.05 \mathrm{NS}$ \\
\hline & Nonworking & 225 & 198 & & \\
\hline \multirow[t]{4}{*}{ Family income } & $\leq 5,000$ INR & 62 & 55 & 1.638 & $0.654 p>0.05 \mathrm{NS}$ \\
\hline & $5,001-10,000$ INR & 94 & 83 & & \\
\hline & $10,001-15,000$ INR & 45 & 51 & & \\
\hline & $>15,000$ INR & 61 & 49 & & \\
\hline \multirow{2}{*}{ Parity } & 1 & 130 & 104 & 4.309 & $0.139 p>0.05 \mathrm{NS}$ \\
\hline & 2 and more & 132 & 134 & & \\
\hline \multirow[t]{3}{*}{ Type of delivery } & Normal & 153 & 157 & 6.609 & $0.035 p<0.05 S$ \\
\hline & Cesarean & 109 & 78 & & \\
\hline & Others & 0 & 3 & & \\
\hline \multirow[t]{5}{*}{ Gestational age } & 37 weeks & 49 & 48 & 3.075 & $0.548 p>0.05 \mathrm{NS}$ \\
\hline & 38 weeks & 57 & 42 & & \\
\hline & 39 weeks & 70 & 76 & & \\
\hline & 40 weeks & 76 & 61 & & \\
\hline & More than 40 weeks & 10 & 11 & & \\
\hline \multirow[t]{2}{*}{ Sex of the baby } & Male & 141 & 123 & 1.981 & $0.396 p>0.05 \mathrm{NS}$ \\
\hline & Female & 121 & 115 & & \\
\hline \multirow[t]{2}{*}{ Weight of the baby } & $<2.5 \mathrm{~kg}$ & 46 & 47 & 0.395 & $0.566 p>0.05 \mathrm{NS}$ \\
\hline & $\geq 2.5 \mathrm{~kg}$ & 216 & 191 & & \\
\hline
\end{tabular}

S: Significant; NS: Not significant

Table 5: Association between newborn challenges and demographic variables $(n=500)$

\begin{tabular}{|c|c|c|c|c|c|}
\hline \multirow{2}{*}{ Demographics } & & \multicolumn{2}{|c|}{ Newborn challenges } & \multicolumn{2}{|c|}{ Chi-square } \\
\hline & & $\leq$ median (6) & $>$ median (6) & Value & $p$-value \\
\hline \multirow[t]{5}{*}{ Age (in years) } & $18-20$ & 10 & 0 & 24.537 & $0.000 p<0.05 S$ \\
\hline & $21-24$ & 126 & 29 & & \\
\hline & $25-29$ & 141 & 88 & & \\
\hline & $30-34$ & 50 & 34 & & \\
\hline & 35 and above & 15 & 7 & & \\
\hline \multirow[t]{3}{*}{ Religion } & Hindu & 241 & 91 & 9.839 & $0.007 p<0.05 S$ \\
\hline & Muslim & 89 & 63 & & \\
\hline & Christian & 12 & 4 & & \\
\hline \multirow[t]{6}{*}{ Educational status } & Illiterate & 69 & 48 & 9.211 & $0.026 p<0.05 S$ \\
\hline & $1-7$ & 132 & 63 & & \\
\hline & $8-10$ & 93 & 34 & & \\
\hline & PUC & 48 & 13 & & \\
\hline & Degree & 0 & 0 & & \\
\hline & Postgraduation & 0 & 0 & & \\
\hline \multirow[t]{2}{*}{ Occupation } & Working & 63 & 16 & 5.589 & $0.024 p<0.05 S$ \\
\hline & Nonworking & 279 & 142 & & \\
\hline
\end{tabular}


(Cont'd...)

\begin{tabular}{|c|c|c|c|c|c|}
\hline \multirow{2}{*}{ Demographics } & & \multicolumn{2}{|c|}{ Newborn challenges } & \multicolumn{2}{|c|}{ Chi-square } \\
\hline & & $\leq$ median (6) & >median (6) & Value & $p$-value \\
\hline \multirow[t]{4}{*}{ Family income } & $\leq 5,000$ INR & 81 & 36 & 2.200 & $0.532 p>0.05 \mathrm{NS}$ \\
\hline & $5,001-10,000$ INR & 114 & 63 & & \\
\hline & 10,001-15,000 INR & 69 & 27 & & \\
\hline & $>15,000$ INR & 78 & 32 & & \\
\hline \multirow[t]{2}{*}{ Parity } & 1 & 223 & 11 & 169.572 & $0.000 p<0.05 S$ \\
\hline & 2 and more & 119 & 147 & & \\
\hline \multirow[t]{3}{*}{ Type of delivery } & Normal & 214 & 96 & 1.288 & $0.787 p>0.05 \mathrm{NS}$ \\
\hline & Cesarean & 126 & 61 & & \\
\hline & Others & 2 & 1 & & \\
\hline \multirow[t]{5}{*}{ Gestational age } & 37 weeks & 62 & 35 & 2.653 & $0.622 p>0.05 \mathrm{NS}$ \\
\hline & 38 weeks & 70 & 29 & & \\
\hline & 39 weeks & 96 & 50 & & \\
\hline & 40 weeks & 99 & 38 & & \\
\hline & More than 40 weeks & 15 & 6 & & \\
\hline \multirow[t]{2}{*}{ Sex of the baby } & Male & 181 & 83 & 0.007 & $1.000 p>0.05 \mathrm{NS}$ \\
\hline & Female & 161 & 75 & & \\
\hline \multirow[t]{2}{*}{ Weight of the baby } & $<2.5 \mathrm{~kg}$ & 68 & 25 & 1.177 & $0.323 p>0.05 \mathrm{NS}$ \\
\hline & $\geq 2.5 \mathrm{~kg}$ & 274 & 133 & & \\
\hline
\end{tabular}

S: Significant; NS: Not significant

Table 6: Association between physiological challenges and demographic variables $(n=500)$

\begin{tabular}{|c|c|c|c|c|c|}
\hline \multirow{2}{*}{ Demographics } & & \multicolumn{2}{|c|}{ Physiological challenges } & \multicolumn{2}{|c|}{ Chi-square } \\
\hline & & $\leq$ median (16) & >median (16) & Value & $p$-value \\
\hline \multirow[t]{5}{*}{ Age (in years) } & $18-20$ & 4 & 6 & 4.659 & $0.327 p>0.05 \mathrm{NS}$ \\
\hline & $21-24$ & 96 & 59 & & \\
\hline & $25-29$ & 120 & 109 & & \\
\hline & $30-34$ & 49 & 35 & & \\
\hline & 35 and above & 12 & 10 & & \\
\hline \multirow[t]{3}{*}{ Religion } & Hindu & 189 & 143 & 0.643 & $0.727 p>0.05 \mathrm{NS}$ \\
\hline & Muslim & 82 & 70 & & \\
\hline & Christian & 10 & 6 & & \\
\hline \multirow[t]{6}{*}{ Educational status } & Illiterate & 0 & 2 & 4.941 & $0.416 p>0.05$ NS \\
\hline & $1-7$ & 59 & 56 & & \\
\hline & $8-10$ & 109 & 86 & & \\
\hline & PUC & 78 & 49 & & \\
\hline & Degree & 23 & 25 & & \\
\hline & Postgraduation & 2 & 1 & & \\
\hline \multirow[t]{2}{*}{ Occupation } & Working & 37 & 40 & 4.771 & $0.061 p<0.05 S$ \\
\hline & Nonworking & 244 & 179 & & \\
\hline \multirow{4}{*}{ Family income } & $\leq 5,000$ INR & 71 & 46 & 16.442 & $0.001 p<0.05 S$ \\
\hline & $5,001-10,000$ INR & 93 & 84 & & \\
\hline & $10,001-15,000$ INR & 41 & 55 & & \\
\hline & $>15,000$ INR & 76 & 34 & & \\
\hline \multirow[t]{2}{*}{ Parity } & 1 & 136 & 98 & 3.462 & $0.263 p>0.05 \mathrm{NS}$ \\
\hline & 2 and more & 145 & 121 & & \\
\hline \multirow[t]{3}{*}{ Type of delivery } & Normal & 170 & 140 & 2.953 & $0.387 p>0.05 N S$ \\
\hline & Cesarean & 109 & 78 & & \\
\hline & Others & 2 & 1 & & \\
\hline \multirow[t]{5}{*}{ Gestational age } & 37 weeks & 62 & 35 & 4.235 & $0.378 p>0.05 \mathrm{NS}$ \\
\hline & 38 weeks & 50 & 49 & & \\
\hline & 39 weeks & 78 & 68 & & \\
\hline & 40 weeks & 79 & 58 & & \\
\hline & More than 40 weeks & 12 & 9 & & \\
\hline \multirow[t]{2}{*}{ Sex of the baby } & Male & 153 & 111 & 0.972 & $0.712 p>0.05 \mathrm{NS}$ \\
\hline & Female & 128 & 108 & & \\
\hline \multirow[t]{2}{*}{ Weight of the baby } & $<2.5 \mathrm{~kg}$ & 55 & 38 & 0.401 & $0.564 p>0.05 \mathrm{NS}$ \\
\hline & $\geq 2.5 \mathrm{~kg}$ & 226 & 131 & & \\
\hline
\end{tabular}

S: Significant; NS: Not significant 
Table 7: Association between coping strategies and demographic variables $(n=500)$

\begin{tabular}{|c|c|c|c|c|c|}
\hline \multirow{2}{*}{ Demographics } & & \multicolumn{2}{|c|}{ Coping strategies } & \multicolumn{2}{|c|}{ Chi-square } \\
\hline & & $\leq$ median (19) & >median (19) & Value & $p$-value \\
\hline \multirow[t]{2}{*}{ Occupation } & Working & 33 & 43 & 7.801 & $0.020 p<0.05 S$ \\
\hline & Nonworking & 236 & 188 & & \\
\hline \multirow[t]{5}{*}{ Gestational age } & 37 weeks & 59 & 37 & 9.788 & $0.04 p<0.05 S$ \\
\hline & 38 weeks & 44 & 55 & & \\
\hline & 39 weeks & 72 & 74 & & \\
\hline & 40 weeks & 84 & 54 & & \\
\hline & More than 40 weeks & 10 & 11 & & \\
\hline
\end{tabular}

S: Significant

Table 8: Levene's test for equality of variance among primi- and multimothers

\begin{tabular}{|c|c|c|c|c|c|c|c|}
\hline Challenges & Parity & $n$ & Mean & $\begin{array}{l}\text { Standard } \\
\text { deviation }\end{array}$ & $\begin{array}{l}\text { Standard error } \\
\text { of the mean }\end{array}$ & $t$-value & $p$-value \\
\hline \multirow[t]{2}{*}{ Physiological total } & 1 & 234 & 15.09 & 4.625 & 0.302 & 1.284 & 0.200 \\
\hline & 2 & 266 & 15.68 & 5.660 & 0.350 & & \\
\hline \multirow[t]{2}{*}{ Emotional total } & 1 & 234 & 54.02 & 7.988 & 0.522 & 0.442 & 0.659 \\
\hline & 2 & 266 & 54.34 & 7.850 & 0.485 & & \\
\hline \multirow[t]{2}{*}{ Environmental total } & 1 & 234 & 13.02 & 2.528 & 0.165 & 1.169 & 0.243 \\
\hline & 2 & 266 & 13.30 & 2.779 & 0.172 & & \\
\hline \multirow[t]{2}{*}{ Financial total } & 1 & 234 & 7.45 & 2.719 & 0.178 & 1.578 & 0.115 \\
\hline & 2 & 266 & 7.06 & 2.798 & 0.173 & & \\
\hline \multirow[t]{2}{*}{ Diet total } & 1 & 234 & 8.82 & 2.510 & 0.164 & 0.223 & 0.824 \\
\hline & 2 & 266 & 8.87 & 2.807 & 0.173 & & \\
\hline \multirow[t]{2}{*}{ Breastfeeding total } & 1 & 234 & 9.35 & 2.182 & 0.143 & 5.316 & $0.000 p<0.05 S$ \\
\hline & 2 & 266 & 10.34 & 1.960 & 0.121 & & \\
\hline \multirow[t]{2}{*}{ Communication total } & 1 & 234 & 8.26 & 2.963 & 0.194 & 0.276 & 0.783 \\
\hline & 2 & 266 & 8.19 & 2.970 & 0.183 & & \\
\hline \multirow[t]{2}{*}{ Newborn total } & 1 & 234 & 3.94 & 1.676 & 0.110 & 17.545 & $0.000 p<0.05 S$ \\
\hline & 2 & 266 & 6.89 & 2.058 & 0.127 & & \\
\hline \multirow[t]{2}{*}{ Employment status total } & 1 & 234 & 1.52 & 2.357 & 0.154 & 3.419 & $0.001 p<0.05 S$ \\
\hline & 2 & 266 & 0.85 & 1.929 & 0.119 & & \\
\hline \multirow[t]{2}{*}{ Family support total } & 1 & 234 & 8.17 & 1.262 & 0.083 & 0.811 & 0.418 \\
\hline & 2 & 266 & 8.07 & 1.542 & 0.095 & & \\
\hline \multirow[t]{2}{*}{ Coping total } & 1 & 234 & 19.10 & 3.587 & 0.235 & 0.425 & 0.671 \\
\hline & 2 & 266 & 18.95 & 3.917 & 0.242 & & \\
\hline \multirow[t]{2}{*}{ Breastfeeding total } & 1 & 234 & 9.35 & 2.182 & 0.143 & 5.316 & $0.000 p<.05 S$ \\
\hline & 2 & 266 & 10.34 & 1.960 & 0.121 & & \\
\hline \multirow[t]{2}{*}{ Newborn total } & 1 & 234 & 3.94 & 1.676 & 0.110 & 17.545 & $0.000 p<.05 S$ \\
\hline & 2 & 266 & 6.89 & 2.058 & 0.127 & & \\
\hline \multirow[t]{2}{*}{ Employment status total } & 1 & 234 & 1.52 & 2.357 & 0.154 & 3.419 & $0.001 p<.05 S$ \\
\hline & 2 & 266 & 0.85 & 1.929 & 0.119 & & \\
\hline
\end{tabular}

S: Significant

\section{CONCLUSION}

Findings of the study revealed that majority of the women had a positive adaptation toward various postnatal challenges, and also they were able to cope well during the postpartum period. It may be assumed that in Indian settings the support system of the family members is relatively sound, thus indicating that majority of women are able to cope up with the postpartum challenges. Family members, especially the mothers and the grandmother of the postnatal woman, play a major role in bringing about a positive feeling toward motherhood..$^{5-8}$

\section{REFERENCES}

1. Family centered maternity and newborn care, National guidelines. Early postpartum care of the mother and infant and transition to the community. Available from: www.phac-aspc.gc.ca/ hp-ps/dca-dea/publications/fcm-smp/fcmc-smpf-06-eng.php.

2. Jomeen J. The importance of assessing psychological status during pregnancy, childbirth and the postnatal period as a 
multidimensional construct: a literature review. Clin Eff Nurs 2004 Sep-Dec;8(3-4):143-155.

3. Piliteri, A. Maternal and child health nursing. 4th ed. Philadelphia (PA): Lippincott Williams and Wilkins; 2003.

4. Fraser, DM.; Cooper, MA. Myles textbook for midwives. 15th ed. Philadelphia (PA): Churchill Livingstone; 2009.

5. Hockenberry, W. Nursing care of infants and children. 8th ed. New Delhi: Elsevier Publishers; 2007.
6. Nichols-Francine, H.; Humenick, SS. Child birth education. 2nd ed. Philadelphia (PA): W.B. Saunders; 2000.

7. Morton, U. Mother to mother; birth and postpartum support service, Inc. Fargo: NPPES National Plan and Enumeration System; 1996.

8. Ngai FW, Chan SW. Stress, maternal role competence and satisfaction among Chinese women in the perinatal period. Res Nurs Health 2012 Feb;35(1):30-39. 